\title{
Estimation of a Spearman-Type Multivariate Measure of Local Dependence
}

\author{
Sumaia A. Latif ${ }^{1} \&$ Pedro A. Morettin ${ }^{2}$ \\ ${ }^{1}$ School of Arts, Science and Humanities, University of São Paulo, São Paulo, Brazil \\ ${ }^{2}$ Institute of Mathematics and Statistics, University of São Paulo, São Paulo, Brazil \\ Correspondence: Pedro A. Morettin, Institute of Mathematics and Statistics, University of São Paulo, São Paulo, \\ Brazil. E-mail: pam@ime.usp.br
}

Received: January 6, 2014 Accepted: February 10, 2014 Online Published: March 20, 2014

doi:10.5539/ijsp.v3n2p1 URL: http://dx.doi.org/10.5539/ijsp.v3n2p1

\begin{abstract}
A multivariate measure of local dependence written in terms of copulas is proposed, which if integrated, coincides with a population version of a multivariate global measure of Spearman's rho. We propose nonparametric estimators of this measure for independent sample data and also for time series data. Some properties of the estimators are derived. Simulations with different copulas and sample sizes were performed to assess the theoretical findings. Empirical applications are given for selected economic indexes of 166 countries and for the returns of the DAX, CAC40 and FTSE indexes.
\end{abstract}

Keywords: local dependence, copula, nonparametric estimation, time series

\section{Introduction}

Global measures of association between two or more random variables are those that result in a single numeric value. On the other hand, measures of local dependence between $d$ random variables (with $d \geq 2$ ) are those calculated on points of a $d$-dimensional grid of variables's support, thus resulting in a $d$-dimensional surface of dependence. In both cases, if the random variables are continuous then some of such measures can be written in terms of copulas, which are well known measures of dependence. It should be noted that the development of the theory of copulas was intensified by the 1990s, and copulas provide a means for the analysis of real problems in finance, economics, risk analysis, pattern recongnition, brain signals and many others areas.

Some familiar measures of global association between two random variables are the linear correlation of Pearson, Spearman's rho, Kendall's tau, Gini's coefficient and the Blomqvist's beta (or medial correlation coefficient), where the last four are also called measures of concordance, which is a form of dependence. For more than two random variables, we can find the extensions of the global measures of concordance of Spearman's rho in Ruymgaart and van Zuijlen (1976), Wolff (1980), Joe (1997), Nelsen (1996, 2002), Úbeda-Flores (2005), Taylor (2007) and Schmid and Schmidt (2007a, 2007b), of Kendall's tau and Gini's coefficient in Nelsen (2002) and Taylor (2007), and of Blomqvist's beta in Nelsen (2002), Úbeda-Flores (2005) and Taylor (2007). Furthermore, some measures of multivariate dependence are studied by Joe (1989), Dhaene, Linders, Schoutens, and Vyncke (2013) and Tasena and Dhompongsa (2013), for example.

Considering measures of local dependence for two random variables we mention copulas and density of copulas (Nelsen, 2006; Joe, 1990), the function of Sibuya (1960), the correlation curve of Bjerve and Doksum (1993) and the measure of Bairamov, Kotz, and Kozubowski (2003), besides the measure J (Sricharan, Hero, \& Rajaratnam, 2011) and the local Gaussian correlation (Tjøstheim \& Hufthammer, 2013), among others. For more than two variables, copulas and density of copulas (Nelsen, 2006, among others) are measures of local dependence that we found in the literature.

The aim of this paper is to propose a multivariate measure of local dependence that is written in terms of copulas, and propose two simple nonparametric methods of estimation.

This paper is organized as follows. In Section 2 we establish some notation and review some concepts and results of copulas. In Section 3 we propose a multivariate measure of local dependence written in terms of copulas, and 
discuss some of its basic properties. The inference is studied in Section 4, where empirical and smoothed nonparametric estimators are suggested for independent samples observed from a random vector. Moreover, one nonparametric smoothed estimator and its weakly convergence is established for data coming from a stationary time series process. For these two types of data (independent and indexed by time), simulations and applications were performed in Section 5 and Section 6, respectively. We finished with some conclusions and suggestions in Section 7.

\section{Background}

In this section we introduce some notation and concepts about copulas.

Let $\mathbf{X}=\left(X_{1}, X_{2}, \ldots, X_{d}\right), d \geq 2$, be a $d$-dimensional vector of real random variables. We denote by $F(\mathbf{x})$ and $F_{i}\left(x_{i}\right)$, respectively, the distribution function of $\mathbf{X}$ and $X_{i}, i=1, \ldots, d$. The generalized inverse of $F_{i}$ is defined by $F_{i}^{-1}\left(u_{i}\right)=\inf \left\{x_{i} \in \mathbb{R} \mid F_{i}\left(x_{i}\right) \geq u_{i}\right\}$, for all $u_{i} \in[0,1], i=1, \ldots, d$, using the convention inf $\emptyset=-\infty$.

In this section we mainly follow Nelsen (2006). Conceptually, a copula can be described as a function which joins or "couples" a multivariate distribution function to its univariate marginal distribution functions. Copulas can be used to construct multivariate distributions or to study some scale-free measures of dependence (Fisher, 1997).

Formally, a $d$-copula is a function $C$ from $I^{d}$ to $I=[0,1]$ with the following properties:

(i) for every $\mathbf{u}$ in $I^{d}, C(\mathbf{u})=0$ if at least one coordinate of $\mathbf{u}$ is 0 , and $C(\mathbf{u})=u_{k}$ if all coordinates of $\mathbf{u}$ are equal 1 except $u_{k}$;

(ii) for every $\mathbf{a}$ and $\mathbf{b}$ in $I^{d}$ such that $a_{i} \leq b_{i}$ for all $i, V_{C}([\mathbf{a}, \mathbf{b}]) \geq 0$, where $V_{C}([\mathbf{a}, \mathbf{b}])$ is the $C$-volume of $[\mathbf{a}, \mathbf{b}]$.

That is, considering the point $\left(F_{1}\left(x_{1}\right), F_{2}\left(x_{2}\right), \ldots, F_{d}\left(x_{d}\right), F\left(x_{1}, x_{2}, \ldots, x_{d}\right)\right)$ in $I^{d+1}$ for each $\left(x_{1}, x_{2}, \ldots, x_{d}\right) \in \overline{\mathbb{R}}^{d}=$ $[-\infty,+\infty]^{d}$, then the mapping from $I^{d}$ to $I$ is a copula. It follows that a copula $C$ is a distribution function on $I^{d}$ with uniformly distributed marginals on $I$.

The theorem of Sklar (1959) is central to the theory of copulas and their multivariate version is given bellow.

Sklar's Theorem Let $F$ be a joint distribution function with margins $F_{i}, i=1,2, \ldots, d$. Then there exists a copula $C$ such that for all $\mathbf{x} \in \overline{\mathbb{R}}^{\mathbf{d}}$,

$$
F\left(x_{1}, x_{2}, \ldots, x_{d}\right)=C\left(F_{1}\left(x_{1}\right), F_{2}\left(x_{2}\right), \ldots, F_{d}\left(x_{d}\right)\right) .
$$

If $F_{i}, i=1,2, \ldots, d$, are continuous, then $C$ is unique; otherwise, $C$ is uniquely determined on Ran $F_{1} \times R a n F_{2} \times \ldots \times$ Ran $F_{d}$, where $R a n F_{k}$ is the range of $F_{k}$. Conversely, if $C$ is a copula and $F_{i}, i=1,2, \ldots$, d are distribution functions, then the function $F$ is a joint distribution function with margins $F_{i}, i=1,2, \ldots, d$.

By this theorem, there exists an unique $d$-copula $C$ given by $F\left(x_{1}, \ldots, x_{d}\right)=C\left(F_{1}\left(x_{1}\right), \ldots, F_{d}\left(x_{d}\right)\right)$ for all $\mathbf{x}=$ $\left(x_{1}, \ldots, x_{d}\right)$ in $\overline{\mathbb{R}}^{d}$ and, conversely, if $C$ is a $d$-copula then the function $F$ is a $d$-dimensional distribution function with margins $F_{1}, \ldots, F_{d}$. That is, the univariate margins $F_{i}, i=1, \ldots, d$ are coupled by the copula $C$ resulting in a multivariate distribution function $F$, or a multivariate distribution $F$ can be decomposed in their univariate margins $F_{i}, i=1, \ldots, d$, and also a dependence structure represented by the copula $C$.

It is observed that for $\mathbf{u}=\left(u_{1}, \ldots, u_{d}\right)$ in $I^{d}$, the copula $C$ of $F$ can be rewritten as $C\left(u_{1}, \ldots, u_{d}\right)=F\left(F_{1}^{-1}\left(u_{1}\right), \ldots\right.$, $\left.F_{d}^{-1}\left(u_{d}\right)\right)$.

The next result gives bounds (called Fréchet-Hoeffding bounds) for any copula $C$ :

$$
W(\mathbf{u}) \leq C(\mathbf{u}) \leq M(\mathbf{u}),
$$

where $W=\max \left(u_{1}+u_{2}+\ldots+u_{d}-d+1,0\right)$ and $M=\min \left(u_{1}, u_{2}, \ldots, u_{d}\right)$. Moreover, $M$ is a copula for $d \geq 2$ whereas $W$ is a copula for $d=2$ but it is not for any $d \geq 3$.

Other copula commonly used is the product copula defined by $\Pi(\mathbf{u})=u_{1} \times \ldots \times u_{d}$.

We now provide some useful properties of copulas. Let $\mathbf{X}=\left(X_{1}, X_{2}, \ldots, X_{d}\right)$ be a $d$-dimensional random vector of continuous values with copula $C$. Then:

(1) if $\alpha_{i}, i=1,2, \ldots, d$ are strictly increasing on $\operatorname{Ran} X_{i}, i=1,2, \ldots, d$, respectively, then the copula of $\left(\alpha_{1}\left(X_{1}\right)\right.$, $\left.\alpha_{2}\left(X_{2}\right), \ldots, \alpha_{d}\left(X_{d}\right)\right)$ is equal to $C$. That is, $C$ is invariant under strictly increasing transformations of $X_{i}, i=$ $1,2, \ldots, d$;

(2) $X_{1}, X_{2}, \ldots, X_{d}$ are independent if and only if $C=\Pi$; 
(3) $\left(X_{1}, X_{2}, \ldots, X_{d}\right)$ is comonotonic if and only if $C=M$; that is, each of the random variables $X_{1}, X_{2}, \ldots, X_{d}$ is almost surely a strictly increasing function of any of the others if and only if $C=M$; this can be interpreted as the extension of the concept of perfect positive correlation;

(4) if $C_{1}$ and $C_{2}$ are copulas, we say that $C_{1}$ is smaller (or less concordant) than $C_{2}$, denoted by $C_{1} \prec_{C} C_{2}$, if $C_{1}(\mathbf{u}) \leq C_{2}(\mathbf{u})$ and $\bar{C}_{1}(\mathbf{u}) \leq \bar{C}_{2}(\mathbf{u})$, for all $\mathbf{u}$ in $I^{d}$ (here, $\bar{C}_{i}=P_{i}[\mathbf{U}>\mathbf{u}]$ denotes the survival function). This is because we want to assess whether $X_{1}, X_{2}, \ldots, X_{d}$ are all both "large simultaneously" and "small simultaneously". For example, $W$ is smaller than every copula, and $M$ is larger than every copula. This partial ordering of the set of copulas is called the concordance ordering;

(5) the $d$-volume between the graphs of $M$ and $\Pi$ and of $W$ and $\Pi$ are $a_{d}=\iint \ldots \int_{I^{d}}[M(\mathbf{u})-\Pi(\mathbf{u})] d u_{1} d u_{2} \ldots d u_{d}=$ $\frac{1}{d+1}-\frac{1}{2^{d}}$ and $b_{d}=\iint \ldots \int_{I^{d}}[W(\mathbf{u})-\Pi(\mathbf{u})] d u_{1} d u_{2} \ldots d u_{d}=\frac{1}{(d+1) !}-\frac{1}{2^{d}}$, respectively, and hence $\lim _{d \rightarrow \infty} \frac{b_{d}}{a_{d}}=0$. That is, as $d$ increases, the graphs of $W(\mathbf{u})$ and $\Pi(\mathbf{u})$ are much closer to each other than those of $M(\mathbf{u})$ and $\Pi(\mathbf{u})$. The equality between $a_{d}$ and $b_{d}$ occur only for $d=2$ (Nešlehová, 2004).

\section{Spearman-Type Multivariate Measure of Local Dependence}

From the Inequality (1) we have that

$$
\frac{W(\mathbf{u})-\Pi(\mathbf{u})}{M(\mathbf{u})-\Pi(\mathbf{u})} \leq \frac{C(\mathbf{u})-\Pi(\mathbf{u})}{M(\mathbf{u})-\Pi(\mathbf{u})} \leq 1,
$$

from which, we propose a local measure of dependence

$$
\delta(\mathbf{u})=\frac{C(\mathbf{u})-\Pi(\mathbf{u})}{M(\mathbf{u})-\Pi(\mathbf{u})},
$$

for all $\mathbf{u} \in(0,1)^{d}$, which can be interpreted as a type of normalized version of the difference between copula and product copula.

This suggestion can be further supported considering two multivariate global measures of Spearman's rho. Firstly, Schmid and Schmidt (2007a) provide the following representation for the bivariate global measure of Spearman's rho

$$
\rho=\frac{\operatorname{Cov}(U, V)}{\sqrt{\operatorname{Var}[U] \operatorname{Var}[V]}}=\frac{\int_{[0,1]^{2}}(C(u, v)-\Pi(u, v)) d u d v}{\int_{[0,1]^{2}}(M(u, v)-\Pi(u, v)) d u d v},
$$

and also their straightforward $d$-variate extension

$$
\rho=\frac{\int_{[0,1]^{d}}(C(\mathbf{u})-\Pi(\mathbf{u})) d \mathbf{u}}{\int_{[0,1]^{d}}(M(\mathbf{u})-\Pi(\mathbf{u})) d \mathbf{u}},
$$

which can be considered locally if we abandon the aspect of volume in the numerator and denominator represented by the integration.

Secondly, the conditional multivariate version of Spearman's rho was studied by Schmid and Schmidt (2007b), and it is given by

$$
\rho=\frac{\int_{[0,1]^{d}}(C(\mathbf{u})-\Pi(\mathbf{u})) g(\mathbf{u}) d \mathbf{u}}{\int_{[0,1]^{d}}(M(\mathbf{u})-\Pi(\mathbf{u})) g(\mathbf{u}) d \mathbf{u}},
$$

where $g \geq 0$ is some measurable weighting function such that the integrals exist. In our analysis, $g$ can be thought as having positive weight only on $\mathbf{u} \pm \epsilon=\left(\left[u_{1}-\epsilon_{1} ; u_{1}+\epsilon_{1}\right], \ldots,\left[u_{d}-\epsilon_{d} ; u_{d}+\epsilon_{d}\right]\right) \subset(0,1)^{d}$ with $\epsilon_{i}>0$, such that $\epsilon_{i} \rightarrow 0$ for $i=1,2, \ldots, d$, and zero weight otherwise. This concept describes a measure evaluated locally around $\mathbf{u}$.

In what follows, we give some properties of the local measure $\delta(\mathbf{u})$ for each $\mathbf{u} \in(0,1)^{d}$ :

(1) $\delta(\mathbf{u})$ is defined for every random vector whose components are all continuous;

(2) $\frac{W(\mathbf{u})-\Pi(\mathbf{u})}{M(\mathbf{u})-\Pi(\mathbf{u})} \leq \delta(\mathbf{u}) \leq 1$;

(3) $C(\mathbf{u})=\Pi(\mathbf{u})$ if and only if $\delta(\mathbf{u})=0$;

(4) $C(\mathbf{u})=M(\mathbf{u})$ if and only if $\delta(\mathbf{u})=1$; 
(5) if $\alpha_{i}, i=1,2, \ldots, d$, are strictly increasing functions of $X_{1}, X_{2}, \ldots, X_{d}$, denoted by $\alpha(\mathbf{X})$, then $\delta$ calculated on both $\alpha(\mathbf{X})$ and $\mathbf{X}$ are the same;

(6) if $C_{n}(\mathbf{u}) \rightarrow C(\mathbf{u})$ uniformly, then $\delta_{C_{n}}(\mathbf{u}) \rightarrow \delta_{C}(\mathbf{u})$ as $n \rightarrow \infty$.

As an example, the behavior of the multivariate measure of local dependence given by Formula (2), considering $d=3$ and Gaussian copula with parameters $(0.723,0.702,0.704)$, is shown in Figure 1 , which is composed by one bivariate contour plot for each of the following values of the third component: $0.05,0.25,0.50,0.75$ and 0.95 . It can be seen that as the third component goes from 0.05 to 0.95 , the greater dependence (contour curves with value 0.8 , for example) moves from the upper right quadrant to the both sides of the main diagonal.
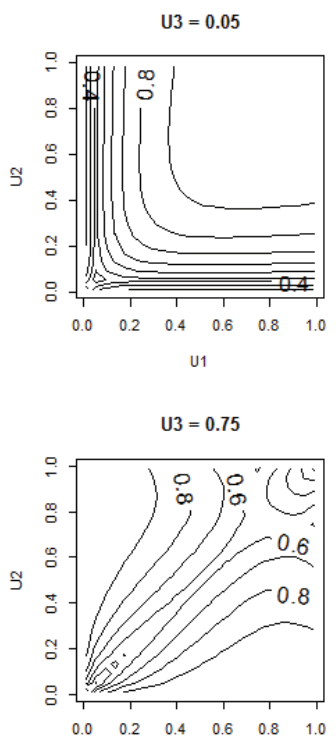

U1
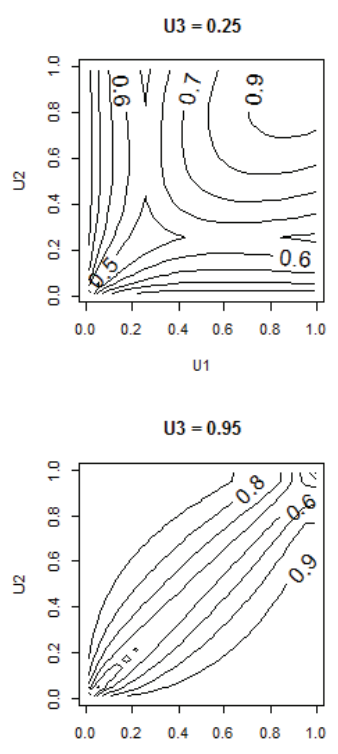

U1

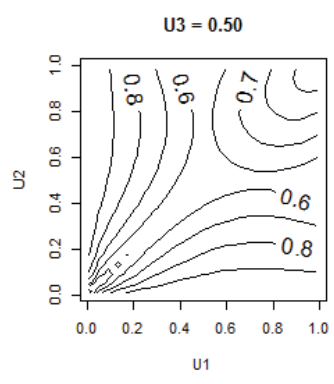

Figure 1. Contour plots for some levels $(0.05,0.25,0.50,0.75$ and 0.95$)$ of the third component of the trivariate Spearman-type measure of local dependence $\delta$ (Formula (2)), considering a Gaussian copula with parameters

$(0.723,0.702,0.704)$

\section{Estimation of the Local Measure}

Copulas may be estimated by nonparametric, semiparametric and parametric methods, and so the same holds for the proposed measure. As it is known, for the last two methods we firstly must choose a suitable family of copulas which may not be a simple task, while for the nonparametric methods no copulas's family must be imposed in advance. For this reason, in this section we will deal with methods that use the empirical copula or copula smoothed by kernel for data observed independently and over time.

\subsection{Independent Sample Data}

Let $\left\{\mathbf{X}_{j}=\left(X_{1 j}, \ldots, X_{d j}\right), j=1, \ldots, n\right\}$ be a random sample of size $n$ observed from the continuous random vector $\mathbf{X}$.

Consider the following empirical estimators of the joint and marginal distributions of $X_{1}, \ldots, X_{d}$ :

$$
F_{n}\left(x_{1}, \ldots, x_{d}\right)=\frac{1}{n} \sum_{j=1}^{n} I\left(X_{1 j} \leq x_{1}, \ldots, X_{d j} \leq x_{d}\right),
$$

and

$$
F_{\text {in }}\left(x_{i}\right)=\frac{1}{n} \sum_{j=1}^{n} I\left(X_{i j} \leq x_{i}\right), i=1, \ldots, d .
$$

Then, the empirical copula is given by $C_{n}(\mathbf{u})=F_{n}\left(F_{1 n}^{-1}\left(u_{1}\right), \ldots, F_{d n}^{-1}\left(u_{d}\right)\right), \forall(\mathbf{u}) \in[0,1]^{d}$, where $F_{\text {in }}^{-1}\left(u_{i}\right)$ are the empirical quantiles with probability levels $u_{i}, i=1, \ldots, d$. 
Thus, using the plug-in method, we obtain the following empirical estimator for $\delta$ :

$$
\delta_{n}(\mathbf{u})=\frac{C_{n}(\mathbf{u})-\Pi(\mathbf{u})}{M(\mathbf{u})-\Pi(\mathbf{u})}, \forall \mathbf{u} \in(0,1)^{d},
$$

where we have excluded the extremes of the $d$-dimensional cube.

The asymptotic behavior of the copula process $\sqrt{n}\left(C_{n}(\mathbf{u})-C(\mathbf{u})\right)$ was investigated by Rüchendorf (1976), Stute (1984), GänBler and Stute (1987) and Fermanian, Radulovic, and Wegkamp (2004), besides Tsukahara (2005), Schmid and Schmidt (2007b) and Segers (2012), for example.

The following theorem can be found in Schmid and Schmidt (2007b), and it was used in the proof of Theorem 2, that brings the weak convergence of the estimator given by Formula (3).

Theorem 1 Let $F$ be a continuous d-dimensional distribution function with copula $C$. Under the additional assumption that the ith partial derivatives $\partial_{i} C(\mathbf{u})$ exist and are continuous for $i=1, \ldots, d$, we have

$$
\sqrt{n}\left(C_{n}-C\right)(\mathbf{u}) \stackrel{W}{\longrightarrow} \mathbb{G}_{C}(\mathbf{u}), \forall \mathbf{u} \in[0,1]^{d},
$$

where $\mathbb{G}_{C}(\mathbf{u})$ is a centered Gaussian process. Weak convergence takes place in $l^{\infty}\left([0,1]^{d}\right)$ and

$$
\mathbb{G}_{C}(\mathbf{u})=\mathbb{B}_{C}(\mathbf{u})-\sum_{i=1}^{d} \partial_{i} C(\mathbf{u}) \mathbb{B}_{C}\left(\mathbf{u}^{(i)}\right) .
$$

The vector $\mathbf{u}^{(i)}$ denotes the vector where all coordinates, except the ith coordinate of $\mathbf{u}$, are replaced by 1 . The process $\mathbb{B}_{C}$ is a tight centered Gaussian process on $[0,1]^{d}$ with covariance function

$$
E\left[\mathbb{B}_{C}(\mathbf{u}) \mathbb{B}_{C}(\mathbf{v})\right]=C(\mathbf{u} \wedge \mathbf{v})-C(\mathbf{u}) C(\mathbf{v}),
$$

i.e., $\mathbb{B}_{C}$ is a d-dimensional Brownian bridge.

Theorem 2 Under the assumptions and notation of Theorem 1,

$$
\sqrt{n}\left(\delta_{n}-\delta\right)(\mathbf{u}) \stackrel{W}{\longrightarrow} \mathbb{G}_{\delta}(\mathbf{u}), \forall \mathbf{u} \in(0,1)^{d}
$$

where $\mathbb{G}_{\delta}(\mathbf{u})$ is a centered Gaussian process given by

$$
\mathbb{G}_{\delta}(\mathbf{u})=\frac{1}{g(\mathbf{u})}\left(\mathbb{B}_{C}(\mathbf{u})-\sum_{i=1}^{d} \partial_{i} C(\mathbf{u}) \mathbb{B}_{C}\left(\mathbf{u}^{(i)}\right)\right),
$$

with $g(\mathbf{u})=M(\mathbf{u})-\Pi(\mathbf{u})$.

Proof. Under the assumptions that $F$ is a continuous $d$-dimensional distribution function with copula $C$ and such that the ith partial derivatives of $\partial_{i} C(\mathbf{u})$ exist and are continuous for $i=1, \ldots, d$, Theorem 1 is valid. Because $\delta(\mathbf{u})$ (Formula (2)) can be obtained as $C \mapsto\left(C, C_{1}, \ldots, C_{d}\right) \mapsto C \circ\left(C_{1}, \ldots, C_{d}\right)$, where $C_{j}=C\left(1, \ldots, u_{j}, \ldots, 1\right)=u_{j}$, for $j=1,2, \ldots, d$, we can see that the first map is linear and continuous, hence Hadamard-differentiable (see the proof of Lemma 3.9.38 in van der Vaart \& Wellner, 1996), and the second map is Hadamard-differentiable on the non null points in the domain of the function (see the section 3.9.4.3 of van der Vaart \& Wellner, 1996). Then, by the functional delta-method, the process of the empirical multivariate measure of local dependence converges weakly to a centered Gaussian process $\mathbb{G}_{\delta}(\mathbf{u})$.

Another estimator, smoothed by kernel functions is given by:

$$
\hat{\delta}(\mathbf{u})=\frac{\hat{C}(\mathbf{u})-\Pi(\mathbf{u})}{M(\mathbf{u})-\Pi(\mathbf{u})}, \forall \mathbf{u} \in(0,1)^{d},
$$

where

$$
\begin{gathered}
\hat{C}(\mathbf{u})=\hat{F}\left(\hat{F}_{1}^{-1}\left(u_{1}\right), \ldots, \hat{F}_{d}^{-1}\left(u_{d}\right)\right), \\
\hat{F}_{j}^{-1}\left(u_{j}\right)=\inf \left\{x_{j}: \hat{F}_{j}\left(x_{j}\right) \geq u_{j}\right\}, j=1, \ldots, d,
\end{gathered}
$$




$$
\begin{gathered}
\hat{F}(\mathbf{x})=\frac{1}{n} \sum_{i=1}^{n} K\left(\frac{x_{1}-X_{1 i}}{h_{1}}, \ldots, \frac{x_{d}-X_{d i}}{h_{d}}\right), \\
\hat{F}_{j}\left(x_{j}\right)=\frac{1}{n} \sum_{i=1}^{n} K_{j}\left(\frac{x_{j}-X_{j i}}{h_{j}}\right), j=1, \ldots, d,
\end{gathered}
$$

with $K(\mathbf{x})=\int_{-\infty}^{x_{1}} \ldots \int_{-\infty}^{x_{d}} k\left(u_{1}, \ldots, u_{d}\right) d u_{1} \ldots d u_{d}$ for $\int \ldots \int k\left(u_{1}, \ldots, u_{d}\right) d u_{1} \ldots d u_{d}=1$ and $k: \mathbb{R}^{d} \rightarrow \mathbb{R}$. Also $K_{j}(w)=$ $\int_{-\infty}^{w} k_{j}(u) d u$ where the real kernel functions $k_{j}(),. j=1, \ldots, d$ are bounded and symmetric such that $\int k_{j}(u) d u=1$. As usual, $h_{j}>0, j=1, \ldots, d$, are bandwidths (functions of $n$ ) such that $h_{j} \rightarrow 0$ as $n \rightarrow \infty, K\left(x_{1}, \ldots, x_{d} ; h_{1}, \ldots, h_{d}\right)=$ $K\left(\frac{x_{1}}{h_{1}}, \ldots, \frac{x_{d}}{h_{d}}\right), k\left(x_{1}, \ldots, x_{d} ; h_{1}, \ldots, h_{d}\right)=\frac{1}{h_{1} \ldots h_{d}} k\left(\frac{x_{1}}{h_{1}}, \ldots, \frac{x_{d}}{h_{d}}\right)$ and $k_{j}\left(u_{j} ; h_{j}\right)=\frac{1}{h_{j}} k_{j}\left(\frac{u_{j}}{h_{j}}\right)$.

Let us assume that the following regularity conditions hold, with $h=h_{1}=\ldots=h_{d}$ for simplicity.

(C1) $F$ has a bounded derivative of order $q$;

(C2) $F_{j}, j=1, \ldots, d$, are Lipschitz;

(C3) $h \rightarrow 0$ as $n \rightarrow \infty$;

(C4) $\lim _{n \rightarrow \infty} \sqrt{n} h^{q}=0$;

(C5) $\int_{\mathbb{R}} \ldots \int_{\mathbb{R}} x_{1}^{k_{1}} \ldots x_{d}^{k_{d}} k\left(x_{1}, \ldots, x_{d}\right) d x_{1} \ldots d x_{d}=0,1 \leq k_{1}+\ldots+k_{d}<q$;

(C6) $\int_{\mathbb{R}} \cdots \int_{\mathbb{R}}\left|x_{1}\right|^{k_{1}} \ldots\left|x_{d}\right|^{k_{d}}\left|k\left(x_{1}, \ldots, x_{d}\right)\right| d x_{1} \ldots d x_{d}<\infty, 1 \leq k_{1}+\ldots+k_{d} \leq q$;

(C7) $\int_{\mathbb{R}} \cdots \int_{\mathbb{R}}\left(\left|x_{1}\right|+\ldots+\left|x_{d}\right|\right) d K\left(x_{1}, \ldots, x_{d}\right)<\infty$.

Theorem 3 Considering (C1) to (C7) valid and C having continuous partial derivatives, then

$$
\left\{W^{C}(\mathbf{u}) \equiv \sqrt{n}(\hat{\delta}-\delta)(\mathbf{u}), \forall \mathbf{u} \in(0,1)^{d}\right\}
$$

converges weakly to a centered Gaussian process $\left\{G_{\delta_{C}}(\mathbf{u}), \forall \mathbf{u} \in(0,1)^{d}\right\}$ in $l^{\infty}\left((0,1)^{d}\right)$.

Proof. Considering (C1) to (C7) valid and $C$ having continuous partial derivatives, then Theorem 10 of Fermanian et al. (2004) is valid for the $d$-dimensional case, that is, the smoothed copula process $\{\sqrt{n}(\hat{C}(\mathbf{u})-C(\mathbf{u})), \forall \mathbf{u} \in$ $\left.[0,1]^{d}\right\}$ converges weakly to a Gaussian process $\left\{G_{C}(\mathbf{u}), \forall \mathbf{u} \in[0,1]^{d}\right\}$, in $l^{\infty}[0,1]^{d}$. By the continuous mapping theorem, we have that the smoothed process $\left\{W^{C}(\mathbf{u}) \equiv \sqrt{n}(\hat{\delta}-\delta)(\mathbf{u}), \forall \mathbf{u} \in(0,1)^{d}\right\}$ converges weakly to a centered Gaussian process $\left\{G_{\delta_{C}}(\mathbf{u}), \forall \mathbf{u} \in(0,1)^{d}\right\}$ in $l^{\infty}\left((0,1)^{d}\right)$.

Table 1. Biases and mean square errors of $\hat{\delta}$ for the Gaussian copula with parameters $(0.723,0.702,0.704)$, calculated from 1,000 independent samples with sizes 250,500 and 1,000

\begin{tabular}{lccccccc}
\hline Grid & $\mathbf{0 . 0 1}$ & $\mathbf{0 . 0 5}$ & $\mathbf{0 . 2 5}$ & $\mathbf{0 . 5 0}$ & $\mathbf{0 . 7 5}$ & $\mathbf{0 . 9 5}$ & $\mathbf{0 . 9 9}$ \\
\hline Actual & 0.135 & 0.236 & 0.414 & 0.502 & 0.524 & 0.441 & 0.351 \\
\hline $\mathbf{n = 2 5 0}$ & & & & & & & \\
Estimated & 0.030 & 0.191 & 0.408 & 0.500 & 0.516 & 0.399 & 0.185 \\
Bias & 0.105 & 0.044 & 0.006 & 0.003 & 0.007 & 0.044 & 0.146 \\
MSE & 0.012 & 0.007 & 0.003 & 0.002 & 0.002 & 0.007 & 0.025 \\
\hline $\mathbf{n = 5 0 0}$ & & & & & & & \\
Estimated & 0.039 & 0.208 & 0.413 & 0.500 & 0.519 & 0.415 & 0.210 \\
Bias & 0.096 & 0.028 & 0.001 & 0.002 & 0.005 & 0.028 & 0.121 \\
MSE & 0.010 & 0.004 & 0.001 & 0.001 & 0.001 & 0.003 & 0.017 \\
\hline $\mathbf{n = 1 , 0 0 0}$ & & & & & & & \\
Estimated & 0.050 & 0.220 & 0.411 & 0.503 & 0.521 & 0.425 & 0.233 \\
Bias & 0.085 & 0.016 & 0.003 & 0.000 & 0.002 & 0.017 & 0.098 \\
MSE & 0.008 & 0.002 & 0.001 & 0.001 & 0.001 & 0.002 & 0.011 \\
\hline
\end{tabular}

\subsection{Time Series Data}

Consider a $d$-variate strictly stationary process with continuous values represented by $\left\{\left(X_{1 t}, \ldots, X_{d t}\right), t \in \mathbb{Z}\right\}$ whose joint distribution, marginal distributions and copula function are represented by $F\left(x_{1}, \ldots, x_{d}\right), F_{1}\left(x_{1}\right), \ldots, F_{d}\left(x_{d}\right)$ and 
$C\left(u_{1}, \ldots, u_{d}\right)$, respectively. Then the measure given by Formula (2) and their properties are also valid here, and it will be denoted by $\delta_{0}(\mathbf{u})$, where the index value 0 represents the contemporary aspect.

A smoothed estimator for this measure is the same of Formula (4) with $\left(X_{1 i}, \ldots, X_{d i}\right), i=1, \ldots, n$ replaced by $\left(X_{1 t}, \ldots, X_{d t}\right), t=1, \ldots, T$, and $\hat{\delta}(\mathbf{u})$ by $\hat{\delta}_{0}(\mathbf{u})$.

The following regularity conditions are assumed, with $h_{*}=\max \left(h_{1}, \ldots, h_{d}\right)$ with $h_{i} \rightarrow 0, i=1, \ldots, d$, as $T \rightarrow \infty$.

(C1) $T h_{*}^{2} \rightarrow 0$ as $T \rightarrow \infty$;

(C1') $T h_{*}^{4} \rightarrow 0$ as $T \rightarrow \infty$, and the $d$-variate kernel $k$ is even;

(C2) the kernel $k$ has a compact support;

(C3) the process $\left(X_{1 t}, \ldots, X_{d t}\right)$ is $\alpha$-mixing with coefficients $\alpha_{T}=o\left(T^{-a}\right)$ for some $a>1$, as $T \rightarrow \infty$;

(C4) the marginal distributions $F_{j}, j=1, \ldots, d$, are continuous differentiable on the intervals $\left[F_{j}^{-1}(a)-\epsilon ; F_{j}^{-1}(b)+\epsilon\right]$, for every $0<a<b<1$ and some $\epsilon>0$, with positive derivatives $f_{j}$. Moreover, the first partial derivatives of $F$ exist and are Lipschitz continuous on the product of these intervals.

Theorem 4 Let $\left\{\left(X_{1 t}, \ldots, X_{d t}\right), t \in \mathbb{Z}\right\}$ be a strictly stationary process with continuous values. With $(C 1)($ or $(C 1$ ') $)$ to $(C 4)$ valid

$$
\left\{W^{C}(\mathbf{u}) \equiv \sqrt{T}\left(\hat{\delta}_{0}-\delta_{0}\right)(\mathbf{u}), \forall \mathbf{u} \in(0,1)^{d}\right\}
$$

converges weakly to a centered Gaussian process in $l^{\infty}\left((0,1)^{d}\right)$.

Proof. Considering (C1) (or (C1')) to (C4) valid, then Theorem 3 of Fermanian and Scaillet (2003) is valid, that is, the process $\left\{\sqrt{T}(\hat{C}(\mathbf{u})-C(\mathbf{u})), \forall \mathbf{u} \in(0,1)^{d}\right\}$ tends weakly to a centered Gaussian process $\left\{G_{C}(\mathbf{u}), \forall \mathbf{u} \in(0,1)^{d}\right\}$, in $l^{\infty}(0,1)^{d}$. By the continuous mapping theorem, the process $\left\{\sqrt{T}\left(\hat{\delta_{0}}-\delta_{0}\right)(\mathbf{u}), \forall \mathbf{u} \in(0,1)^{d}\right\}$ converges weakly to a centered Gaussian process $\left\{G_{\delta_{0 C}}(\mathbf{u}), \forall \mathbf{u} \in(0,1)^{d}\right\}$ in $l^{\infty}\left((0,1)^{d}\right)$.

\section{Simulations}

To investigate the performance of the smoothed estimator (Formula (4)) for independent samples (denoted by $\hat{\delta}$ ) and time series data (denoted by $\hat{\delta}_{0}$ ), we conduct simulations for the trivariate case on some grid points with equal components given by $0.01,0.05,0.25,0.50,0.75,0.95$ and 0.99 , using 1,000 replications of samples with sizes 250 , 500 and 1,000, trivariate multiplicative kernel $k\left(x_{1}, x_{2}, x_{3}\right)=k_{1}\left(x_{1}\right) k_{1}\left(x_{2}\right) k_{1}\left(x_{3}\right)$, Gaussian kernel, and bandwidths of Azzalini (1981) given by $0.5 \sigma n^{-1 / 3}$. The parameter $\sigma$ was estimated by the sample standard deviation. The R package version 3.0.1 was used.

\subsection{Independent Sample Data}

We considered 1,000 replications of independent samples with different sizes for the following trivariate copulas: Gaussian, with null correlations between their components and also with correlations equal to $(0.723,0.702,0.704)$; Student's $t$, with correlations $(0.8,0.5,0.8)$ and 8 degrees of freedom; and Gumbel, with parameter 2.

The simulation results of $\hat{\delta}$ for the Gaussian copula with null correlations, show biases and mean squared errors generally lower than 0.001 . For the Gaussian copula with nonzero correlations, Table 1 presents on each considered grid point, the theoretical and estimated values of the local measure, and the biases and mean squared errors. We notice that the values of these statistics are usually small, except at the end points of the grid and they decrease with the increase of sample size (usual behavior for all studied copulas). Table 2 presents the simulation results for the $t$ copula, where the biases and the mean squared errors are sligthly greater when compared with the previous simulations. For the Gumbel copula (larger dependence on the upper right side of the support), analyzing the results of Table 3, we see that the biases and the mean squared errors are more pronounced mainly on the grid point 0.99 . 
Table 2. Biases and mean square errors of $\hat{\delta}$ for the $t$ copula with correlations $(0.8,0.5,0.8)$ and 8 degrees of freedom, calculated from 1,000 independent samples with sizes 250, 500 and 1,000

\begin{tabular}{lccccccc}
\hline Grid & $\mathbf{0 . 0 1}$ & $\mathbf{0 . 0 5}$ & $\mathbf{0 . 2 5}$ & $\mathbf{0 . 5 0}$ & $\mathbf{0 . 7 5}$ & $\mathbf{0 . 9 5}$ & $\mathbf{0 . 9 9}$ \\
\hline Actual & 0.195 & 0.267 & 0.417 & 0.505 & 0.541 & 0.510 & 0.480 \\
\hline $\mathbf{n = 2 5 0}$ & & & & & & & \\
Estimated & 0.038 & 0.216 & 0.410 & 0.501 & 0.538 & 0.448 & 0.222 \\
Bias & 0.158 & 0.051 & 0.006 & 0.004 & 0.004 & 0.063 & 0.238 \\
MSE & 0.026 & 0.008 & 0.003 & 0.002 & 0.002 & 0.008 & 0.060 \\
\hline $\mathbf{n = 5 0 0}$ & & & & & & & \\
Estimated & 0.052 & 0.239 & 0.413 & 0.501 & 0.536 & 0.466 & 0.255 \\
Bias & 0.144 & 0.027 & 0.003 & 0.004 & 0.005 & 0.044 & 0.206 \\
MSE & 0.022 & 0.004 & 0.002 & 0.001 & 0.001 & 0.004 & 0.045 \\
\hline $\mathbf{n = 1 , 0 0 0}$ & & & & & & & \\
Estimated & 0.067 & 0.247 & 0.415 & 0.504 & 0.540 & 0.486 & 0.288 \\
Bias & 0.129 & 0.019 & 0.001 & 0.001 & 0.001 & 0.025 & 0.173 \\
MSE & 0.018 & 0.003 & 0.001 & 0.000 & 0.000 & 0.002 & 0.031 \\
\hline
\end{tabular}

Table 3. Biases and mean square errors of $\hat{\delta}$ for the Gumbel copula with parameter 2, calculated from 1,000 independent samples with sizes 250,500 and 1,000

\begin{tabular}{lccccccc}
\hline Grid & $\mathbf{0 . 0 1}$ & $\mathbf{0 . 0 5}$ & $\mathbf{0 . 2 5}$ & $\mathbf{0 . 5 0}$ & $\mathbf{0 . 7 5}$ & $\mathbf{0 . 9 5}$ & $\mathbf{0 . 9 9}$ \\
\hline Actual & 0.034 & 0.109 & 0.320 & 0.469 & 0.566 & 0.622 & 0.632 \\
\hline $\mathbf{n = 2 5 0}$ & & & & & & & \\
Estimated & 0.010 & 0.090 & 0.317 & 0.470 & 0.562 & 0.550 & 0.298 \\
Bias & 0.024 & 0.019 & 0.003 & 0.000 & 0.004 & 0.072 & 0.334 \\
MSE & 0.001 & 0.003 & 0.003 & 0.002 & 0.002 & 0.010 & 0.116 \\
\hline $\mathbf{n = 5 0 0}$ & & & & & & & \\
Estimated & 0.013 & 0.100 & 0.317 & 0.467 & 0.564 & 0.572 & 0.347 \\
Bias & 0.021 & 0.010 & 0.003 & 0.002 & 0.002 & 0.050 & 0.285 \\
MSE & 0.001 & 0.002 & 0.001 & 0.001 & 0.001 & 0.005 & 0.084 \\
\hline $\mathbf{n = 1 , 0 0 0}$ & & & & & & & \\
Estimated & 0.016 & 0.103 & 0.319 & 0.470 & 0.565 & 0.593 & 0.393 \\
Bias & 0.018 & 0.007 & 0.001 & 0.000 & 0.001 & 0.029 & 0.238 \\
MSE & 0.001 & 0.001 & 0.001 & 0.001 & 0.001 & 0.002 & 0.059 \\
\hline
\end{tabular}

The behavior of the estimator's distributions on the three central grid points for the above simulations with sample size 1,000 is shown in Figure 2 through normal quantile plots (where the axis's labels $t q$ and $s q$ means theoretical quantiles and sample quantiles) and in Figure 3 through histograms. We conclude that the limiting Gaussian approximation of the estimator looks acceptable for these chosen points. 

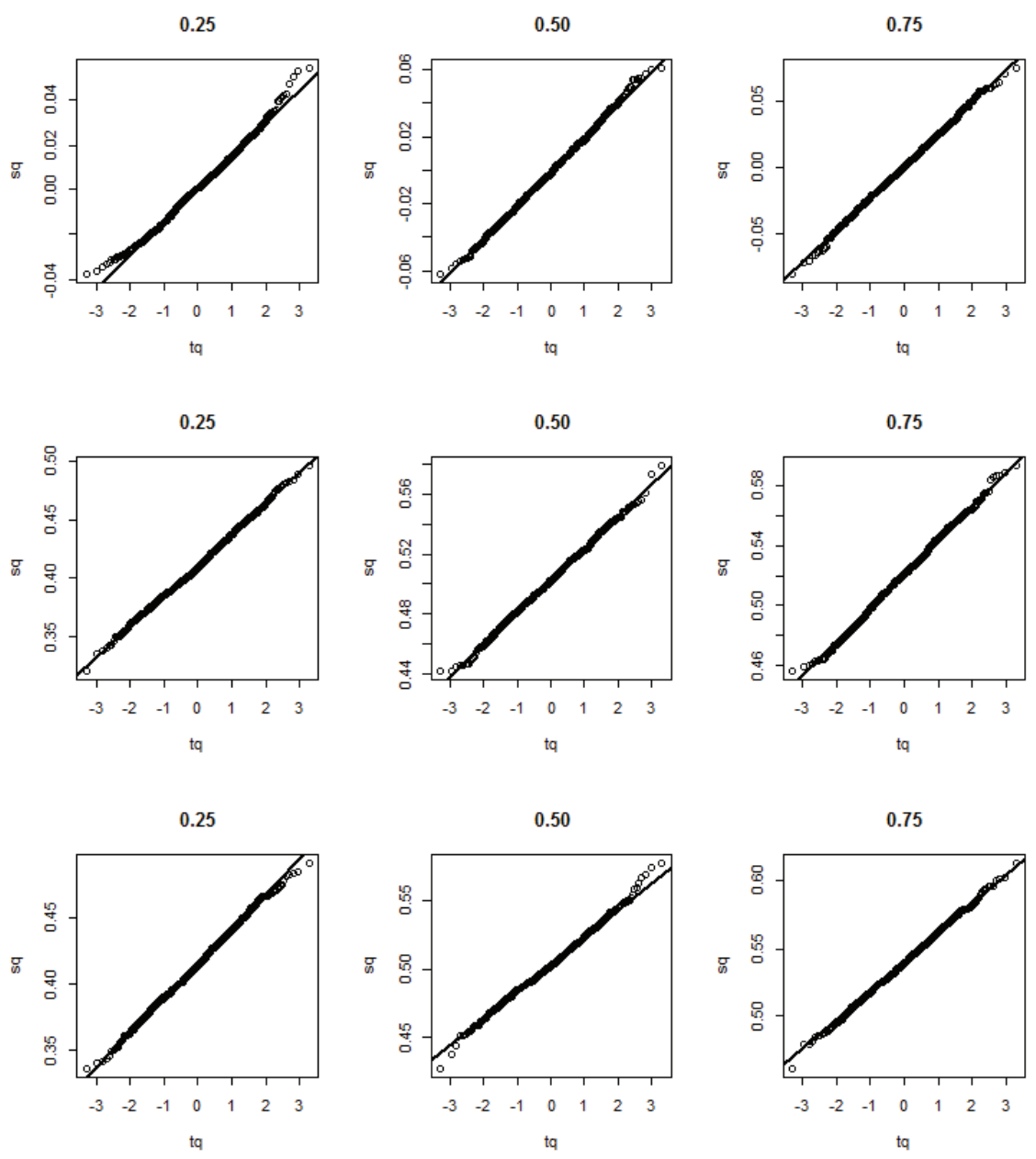

0.25

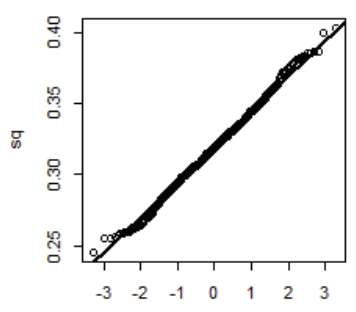

0.50

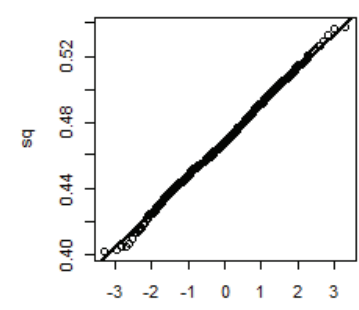

tq

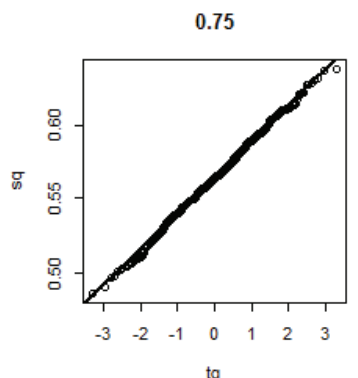

Figure 2. Normal quantile plots of the measure on the grid points $\mathbf{u}=(u, u, u)$ for $u=0.25, u=0.50$ and $u=0.75$ considering simulations with 1,000 samples observed from a Gaussian copula with null correlations, Gaussian copula with correlations equal to $(0.723,0.702,0.704), t$ copula with correlations $(0.8,0.5,0.8)$ and 8 dregrees of freedom, and finally the Gumbel copula with parameter 2 

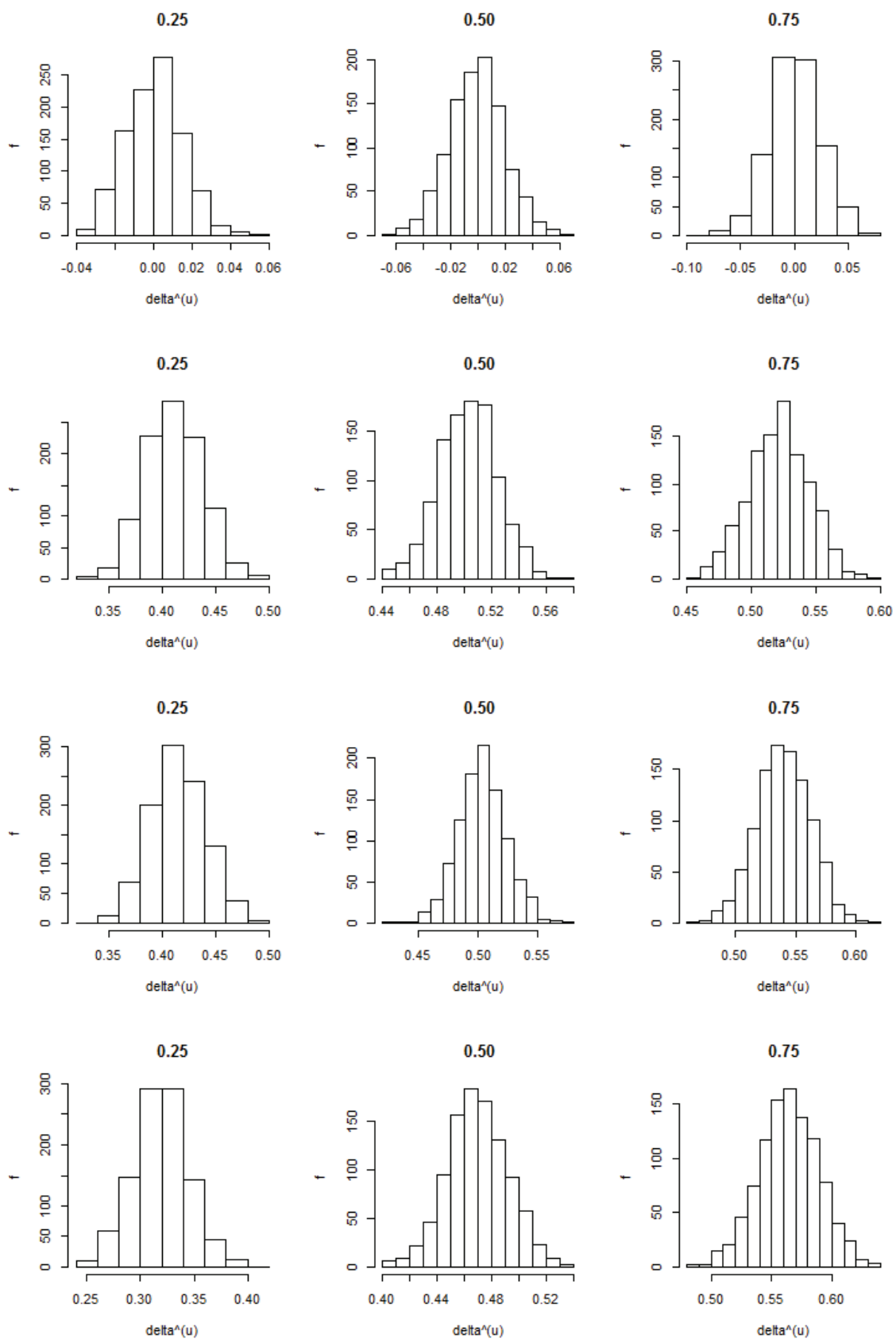

Figure 3. Histograms of the measure on the grid points $\mathbf{u}=(u, u, u)$ for $u=0.25, u=0.50$ and $u=0.75$ considering simulations with 1,000 samples observed from a Gaussian copula with null correlations, Gaussian copula with correlations equal to $(0.723,0.702,0.704), t$ copula with correlations $(0.8,0.5,0.8)$ and 8 degrees of freedom, and finally the Gumbel copula with parameter 2

\subsection{Time Series Data}

Firstly, we consider the trivariate stationary VAR(1) process represented by

$$
\mathbf{Y}_{t}=\boldsymbol{\Phi}_{0}+\boldsymbol{\Phi}_{1} \mathbf{Y}_{t-1}+\epsilon_{t}
$$

where $\mathbf{Y}_{t}=\left(Y_{1 t}, Y_{2 t}, Y_{3 t}\right)^{\prime}, \boldsymbol{\Phi}_{0}=(1,1,1)^{\prime}, \operatorname{vec}\left(\boldsymbol{\Phi}_{1}\right)=(0.3,0,0,0,0.5,0,0,0,0.8)^{\prime}$ and $\epsilon_{t} \sim N(\mathbf{0}, \boldsymbol{\Sigma})$ with vec $(\boldsymbol{\Sigma})=$ $(1.0,0,0,0,1.5,0,0,0,2.1)^{\prime}$. The eigenvalues of $\boldsymbol{\Phi}_{1}$ are in modulus smaller than one and then the Gaussian process referred as $\mathbf{Y}_{t}$ is stationary. Also, the parameters are $\mu=(1.43,2,5)^{\prime}$ and $\operatorname{vec}(\boldsymbol{\Gamma}(0))=(1.1,0,0,0,2,0,0,0,5.83)^{\prime}$ with $\operatorname{vec}(\rho(0))=(1,0,0,0,1,0,0,0,1)^{\prime}$. The behavior of the estimator $\hat{\delta}_{0}$ was very similar to that of Gaussian 
copula with null correlations of the previous subsection, that is, the biases and mean squared errors are very small (generally less than 0.001).

Secondly, we study a trivariate stationary $\operatorname{VAR}(1)$ process with correlated components where $\boldsymbol{\Phi}_{0}=(1,1,1)^{\prime}$, $\operatorname{vec}\left(\boldsymbol{\Phi}_{1}\right)=(0.3,0.1,0.1,0.1,0.5,0.1,0.1,0.1,0.8)^{\prime}$ (eigenvalues equal to $0.86,0.49$ and 0.25$)$ and $\operatorname{vec}(\boldsymbol{\Sigma})=(1.0,0.7$, $0.8,0.7,1.5,0.9,0.8,0.9,2.1)^{\prime}$. Then, $\mu=(3.33,4.44,8.89)^{\prime}$ and $\operatorname{vec}(\boldsymbol{\Gamma}(0))=(1.58,1.54,2.6,1.54,2.89,3.54$, $2.61,3.54,8.78)^{\prime}$ with $\operatorname{vec}(\rho(0))=(1,0.723,0.702,0.723,1,0.704,0.702,0.704,1)^{\prime}$. In this case, the biases and mean squared errors are generally lower than that of the Gaussian copula with nonzero correlations studied in the previous subsection. See Table 4.

Table 4. Biases and mean square errors of $\hat{\delta_{0}}$ calculated from 1,000 time series data with sizes 250, 500 and 1,000, generated from the VAR $(1)$ model with parameters $\phi_{0}=(1,1,1)^{\prime}, \operatorname{vec}\left(\phi_{1}\right)=(0.3,0.1,0.1,0.1,0.5,0.1,0.1,0.1,0.8)^{\prime}$ and $\operatorname{vec}(\boldsymbol{\Sigma})=(1.0,0.7,0.8,0.7,1.5,0.9,0.8,0.9,2.1)^{\prime}$, considering the Gaussian copula with correlations $(0.723$, $0.702,0.704)^{\prime}$

\begin{tabular}{lccccccc}
\hline Grid & $\mathbf{0 . 0 1}$ & $\mathbf{0 . 0 5}$ & $\mathbf{0 . 2 5}$ & $\mathbf{0 . 5 0}$ & $\mathbf{0 . 7 5}$ & $\mathbf{0 . 9 5}$ & $\mathbf{0 . 9 9}$ \\
\hline Actual & 0.135 & 0.236 & 0.414 & 0.502 & 0.523 & 0.442 & 0.340 \\
\hline $\mathbf{T = 2 5 0}$ & & & & & & & \\
Estimated & 0.081 & 0.209 & 0.405 & 0.490 & 0.510 & 0.411 & 0.256 \\
Bias & 0.054 & 0.027 & 0.010 & 0.013 & 0.016 & 0.030 & 0.074 \\
MSE & 0.023 & 0.014 & 0.005 & 0.003 & 0.004 & 0.012 & 0.036 \\
\hline T=500 & & & & & & & \\
Estimated & 0.099 & 0.220 & 0.404 & 0.494 & 0.516 & 0.425 & 0.300 \\
Bias & 0.035 & 0.016 & 0.010 & 0.008 & 0.007 & 0.015 & 0.030 \\
MSE & 0.018 & 0.008 & 0.002 & 0.002 & 0.002 & 0.006 & 0.021 \\
\hline $\mathbf{T = 1 , 0 0 0}$ & & & & & & & \\
Estimated & 0.119 & 0.226 & 0.410 & 0.499 & 0.519 & 0.432 & 0.314 \\
Bias & 0.016 & 0.010 & 0.004 & 0.003 & 0.004 & 0.008 & 0.017 \\
MSE & 0.011 & 0.004 & 0.001 & 0.001 & 0.001 & 0.003 & 0.013 \\
\hline
\end{tabular}
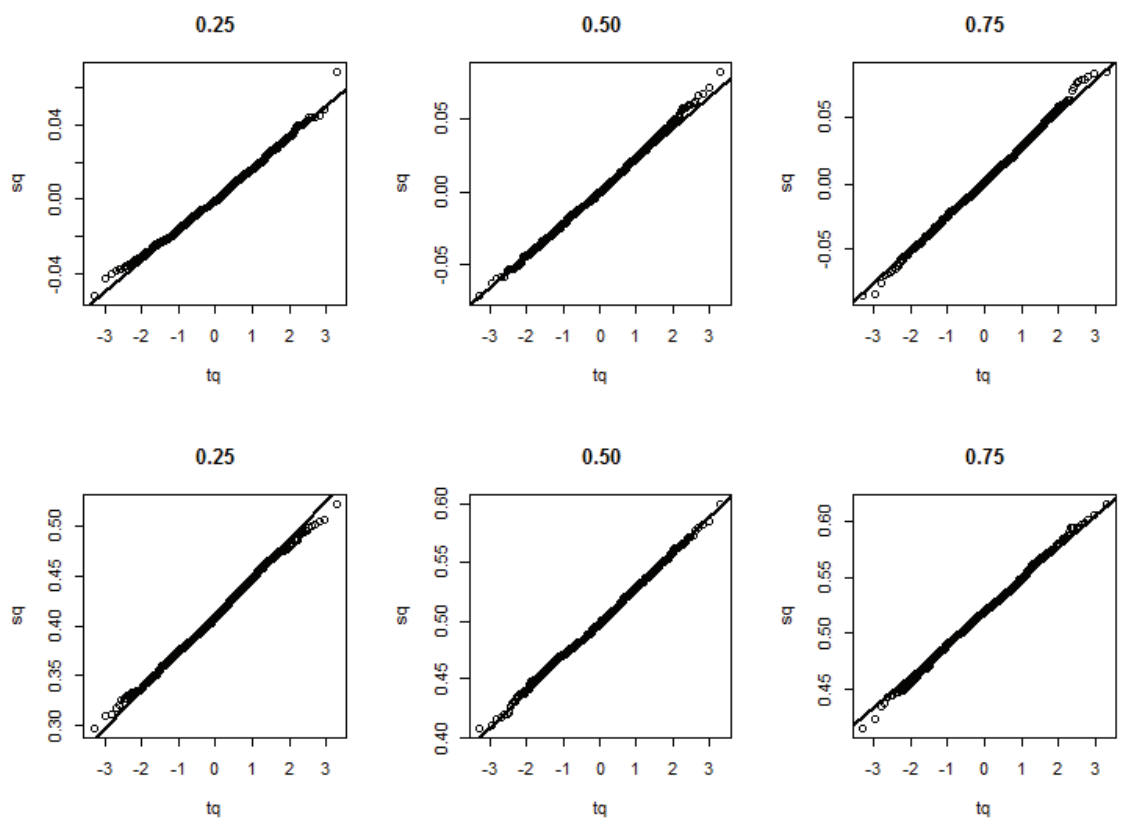

Figure 4. Normal quantile plots of the measure on the grid points $\mathbf{u}=(u, u, u)$ for $u=0.25, u=0.50$ and $u=0.75$ considering simulations with 1,000 time series from a normal distribution with null correlations, and normal distribution with correlations equal to $(0.723,0.702,0.704)$ 

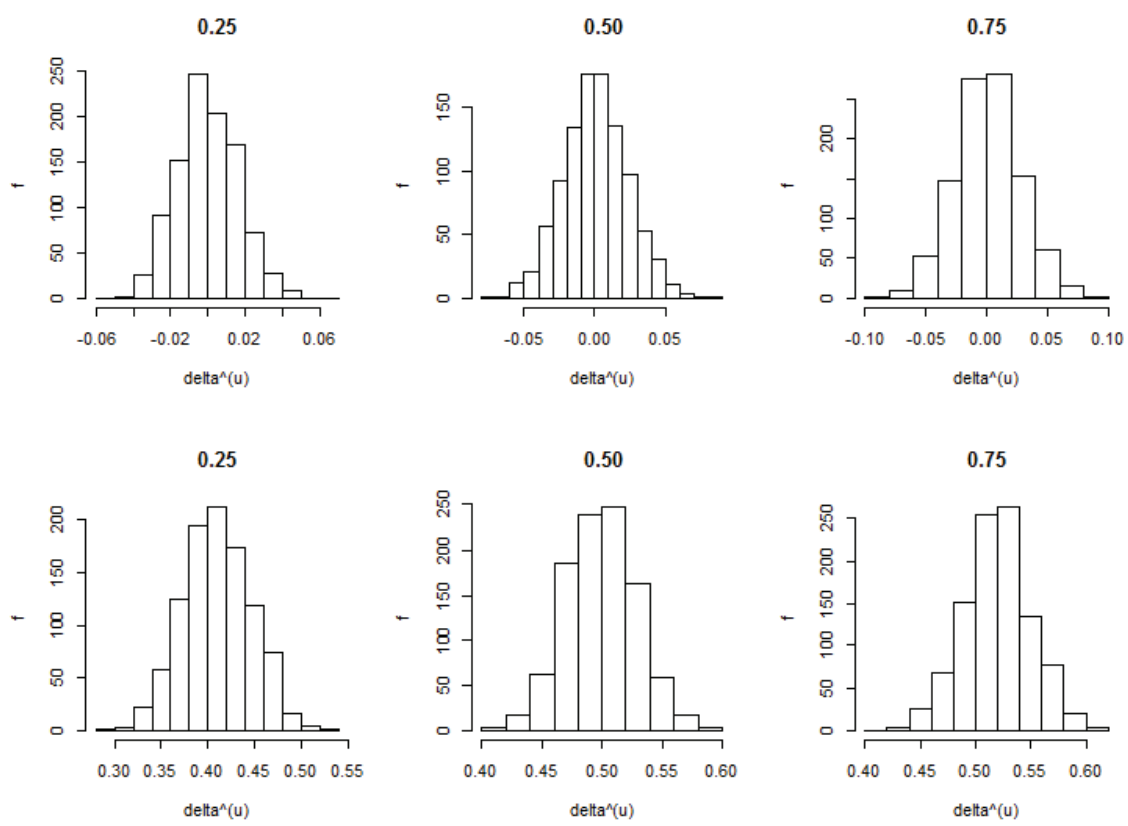

Figure 5. Histograms of the measure on the grid points $\mathbf{u}=(u, u, u)$ for $u=0.25, u=0.50$ and $u=0.75$ considering simulations with 1,000 time series from a normal distribution with null correlations, and normal distribution with correlations equal to $(0.723,0.702,0.704)$

Figures 4 and 5 show some normal quantile plots and histograms for the estimator, showing that the Gaussian approximation is good. We conclude that the results of this section support the corresponding theoretical findings of the previous section.

\section{Applications}

To analyze actual data we use $90 \%$ of the central data (to avoid bad estimations on the boundaries) and bandwidths according to Azzalini (1981) given by $1.3 \sigma n^{-1 / 3}$, although the bandwidth of the previous section brings very similar results for the data considered in this section. As before, the parameter $\sigma$ is estimated by the standard deviation of the samples.

\subsection{Independent Sample Data}

Economic indexes of the GDP (gross domestic product) annual growth rate, the interest rate, and the inflation rate of 166 countries, denoted respectively by $X, Y$ and $Z$, were accessed from the website www.tradingeconomics.com on April 2, 2013.

The scatter plots between the pairs $(X, Y),(X, Z)$ and $(Y, Z)$ are shown at the top panel of Figure 6, where only the last one indicates positive linear relationship, with Pearson's correlation coefficient given by 0.618 . To search for any type of dependence structure between these pairs of variables, we can get their bivariate empirical copula calculating the normalized ranks (ranks divided by $n+1$ instead of $n$, to avoid estimation problems at the boundaries) of $X, Y$ and $Z$, which are denoted by $U_{1}, U_{2}$ and $U_{3}$, respectively. The corresponding scatter plots can be seen at the bottom panel of Figure 6, where the two first show some positive dependence and the latter show an important positive dependence mainly between greater values of the variables. These behaviors were confirmed by the values $0.400,0.314$ and 0.632 of their respective correlation of Spearman's rho.

The estimates of $\hat{\delta}$ are shown in Table 5 for ten grid points with equal components, and the corresponding bivariate local measure estimates on the grid points $0.05,0.25,0.50,0.75$ and 0.95 of the third component $U 3$ are shown in Figure 7, where the local dependence between $U_{1}(X)$ and $U_{2}(Y)$ goes from the upper right side to the bottom right side as $U 3$ goes from 0.05 to 0.95 . That is, when the values of inflation rate are low then the dependence between GDP annual growth rate and interest rate is greater between their higher values, and for high values of inflation rate then the dependence is greater between the higher values of GDP annual growth rate and the lower values of interest rate. 

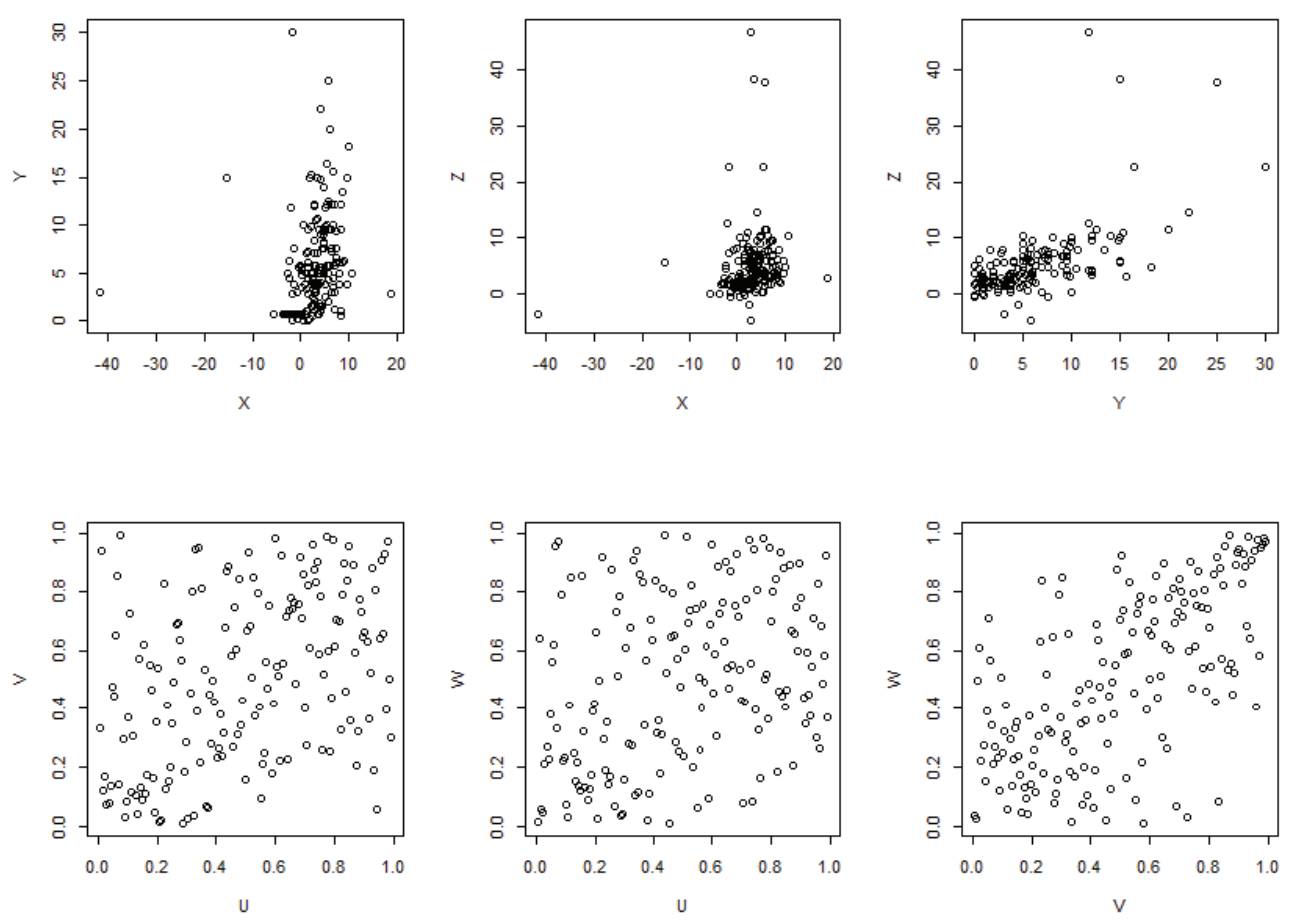

Figure 6. The first three plots are the scatter plots of the GDP annual growth rate $(X)$, the interest rate $(Y)$ and the inflation rate $(Z)$ of 166 countries. The last three plots are scatter plots of their normalized ranks, that is, the bivariate empirical copula densities
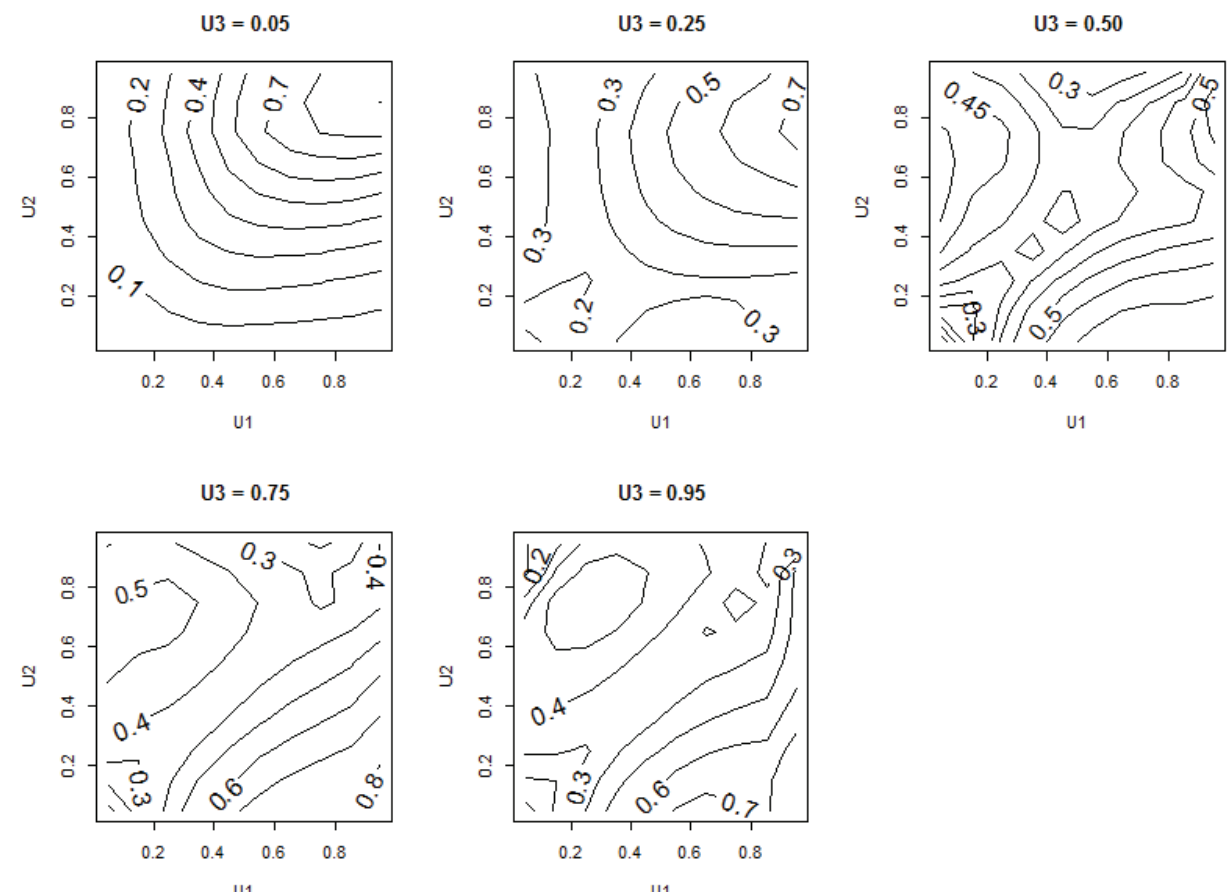

Figure 7. Cconsidering the GDP annual growth rate $(X)$, the interest rate $(Y)$ and the inflation rate $(Z)$ of 166 countries, the estimation of the trivariate Spearman-type measure of local dependence $\hat{\delta}$ (Formula (4)) is showed by bivariate contour plots on some grid points $(0.05,0.25,0.50,0.75,0.95)$ of the third component 
Table 5. Estimates of $\delta$ using the GDP annual growth rate, the interest rate and the inflation rate

\begin{tabular}{lrrrrrrrrrr}
\hline Grid & $\mathbf{0 . 0 5}$ & $\mathbf{0 . 1 5}$ & $\mathbf{0 . 2 5}$ & $\mathbf{0 . 3 5}$ & $\mathbf{0 . 4 5}$ & $\mathbf{0 . 5 5}$ & $\mathbf{0 . 6 5}$ & $\mathbf{0 . 7 5}$ & $\mathbf{0 . 8 5}$ & $\mathbf{0 . 9 5}$ \\
\hline Values & 0.11 & 0.081 & 0.186 & 0.261 & 0.306 & 0.321 & 0.307 & 0.283 & 0.301 & 0.296 \\
\hline
\end{tabular}

\subsection{Time Series Data}

The German, French and English stock indexes abbreviated as DAX, CAC40 and FTSE were observed from January 2nd, 2001 to March 27, 2013 (3,064 observations) in the website www.inventing.com/indexes (on March 28,2013 ), and their log-returns (briefly returns) are represented by $X_{t}, Y_{t}$ and $Z_{t}$ in this order.

Estimates of $\hat{\delta}_{0}$ are given in Table 6 for ten central grid points with equal components, and Figure 8 shows the bivariate scatter plots of $\left(X_{t}, Y_{t}\right),\left(X_{t}, Z_{t}\right)$ and $\left(Y_{t}, Z_{t}\right)$, which exhibit strong positive linear associations. The corresponding coefficients of Pearson's linear correlation are 0.891, 0.820 and 0.900 and of Spearman's rho are 0.893, 0.807 and 0.868 . All of these series are stationary as indicated by the autocorrelation functions in Figure 9 .

Table 6. Estimates of $\delta_{0}$ using the returns of DAX, CAC40 and FTSE from January 2nd, 2001 to March 27, 2013

\begin{tabular}{lrrrrrrrrrr}
\hline Grid & $\mathbf{0 . 0 5}$ & $\mathbf{0 . 1 5}$ & $\mathbf{0 . 2 5}$ & $\mathbf{0 . 3 5}$ & $\mathbf{0 . 4 5}$ & $\mathbf{0 . 5 5}$ & $\mathbf{0 . 6 5}$ & $\mathbf{0 . 7 5}$ & $\mathbf{0 . 8 5}$ & $\mathbf{0 . 9 5}$ \\
\hline Values & 0.549 & 0.623 & 0.631 & 0.634 & 0.651 & 0.669 & 0.686 & 0.699 & 0.697 & 0.697 \\
\hline
\end{tabular}
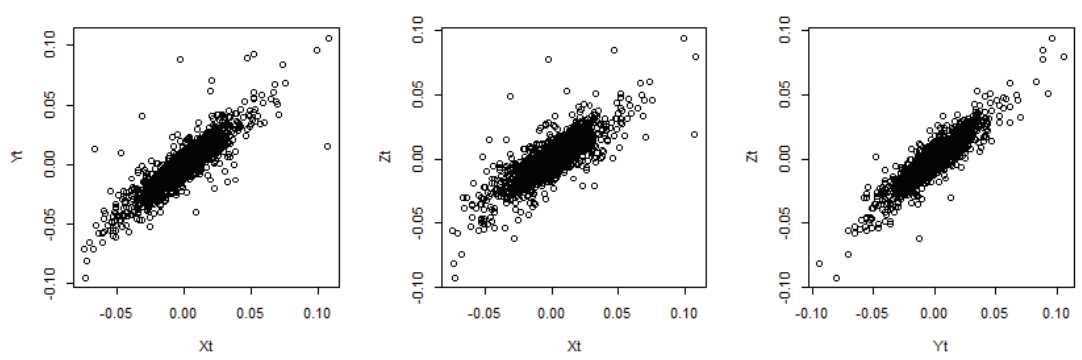

Figure 8. Scatter plots of the returns of the DAX, CAC40 and FTSE stock indexes $\left(X_{t}, Y_{t}\right.$ and $Z_{t}$, in this order) from January 3nd, 2001 to March 27, 2013

$\mathrm{Xt}$

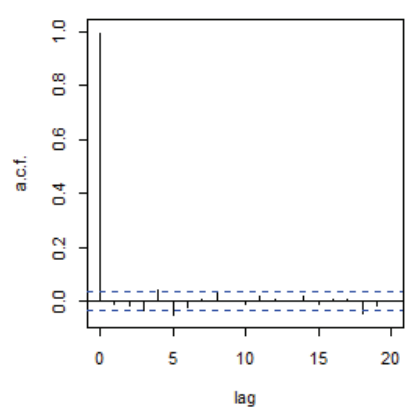

$\mathrm{Yt}_{\mathrm{t}}$

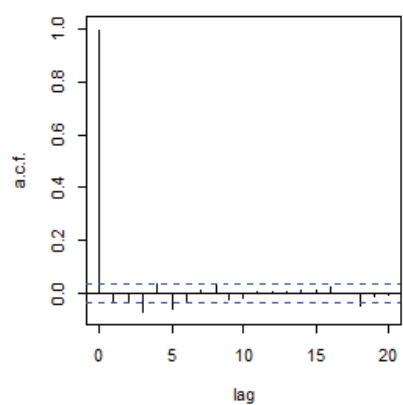

Zt

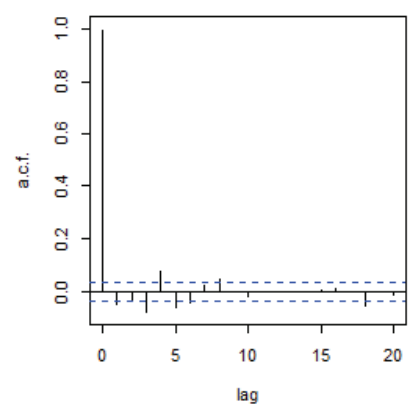

Figure 9. Autocorrelation functions of the returns of the DAX, CAC40 and FTSE stock indexes $\left(X_{t}, Y_{t}\right.$ and $Z_{t}$, in this order)

From Figure 10, we can see that as the grid value of FTSE goes from 0.05 to 0.95 then the local dependence $\left(\hat{\delta}_{0}\right)$ between DAX and CAC40 goes from their higher values to their off-diagonal values. 
$\mathrm{U} 3=0.05$

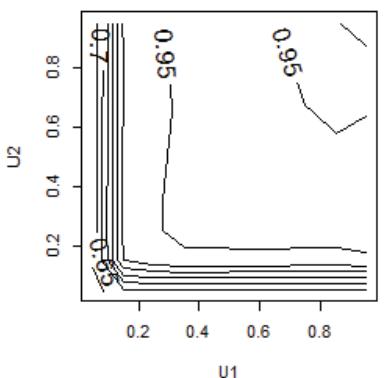

$\mathrm{U} 3=\mathbf{0 . 7 5}$

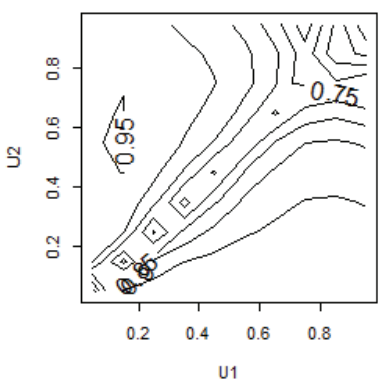

$\mathrm{U} 3=0.25$

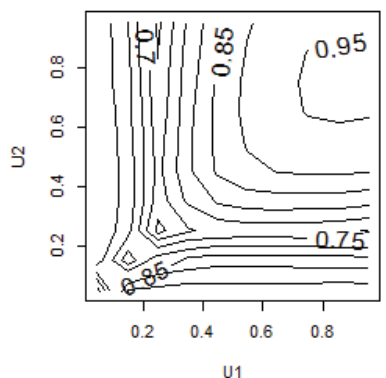

$\mathrm{U} 3 \mathbf{= 0 . 9 5}$

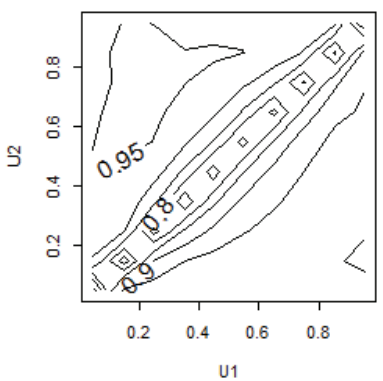

$\mathrm{U} 3=\mathbf{0 . 5 0}$

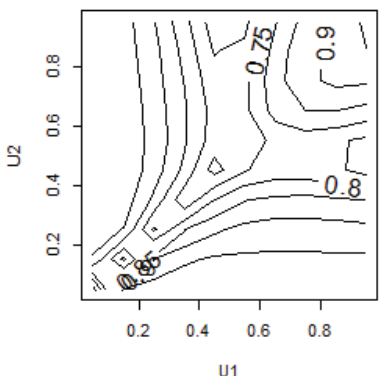

Figure 10. Considering the returns of the DAX, CAC40 and FTSE stock indexes ( $X_{t}, Y_{t}$ and $Z_{t}$, in this order), the estimation of the trivariate Spearman-type measure of local dependence $\hat{\delta}_{0}$ ) (Formula (4)) is showed by bivariate contour plots on some grid points $(0.05,0.25,0.50,0.75,0.95)$ of the third component

\section{Conclusions}

This work proposes a multivariate measure of local dependence, called Spearman-type and denoted by $\delta$, which is derived from the inequality of Fréchet-Hoeffding bounds for copulas, which coincides with the local feature of both a version of the global multivariate measure of Spearman's rho given by Schmid and Schmidt (2007b) and a version of the conditional multivariate measure of Sperman's rho as proposed by Schmid and Schmidt (2007a). It is written in terms of copulas and it satisfies common properties of a measure of dependence, like the upper bound of +1 , the zero value if and only if their components are independent, the scale-invariance and the pointwise convergence.

We suggest an empirical estimator for independent sample data, and a smoothed estimator by kernel for both independent sample data and time series data. For these cases, the weak convergence to a Gaussian process were obtained.

To verify the behavior of the smoothed estimators, we conduct simulations with 1,000 replications and samples of sizes 250, 500 and 1,000, considering the Gaussian, $t$ and Gumbel copulas for independent sample data and Gaussian copulas for time series data. We observe that the biases and the mean squared errors are very small (except on the analyzed grid points 0.01 and 0.99 , which was expected), and their values decrease with the increasing of sample sizes. For samples of size 1,000, the distribution of the estimator is approximately normal except, sometimes, in the lower and/or upper points of the grid.

Applications of the Spearman-type multivariate measure of local dependence to actual data were made using the GDP annual growth rate, interest rate and inflation rate of countries and also using the contemporary returns of the DAX, CAC40 and FTSE from January 3nd, 2001 to March 27, 2013.

Research is needed to propose other estimators with less boundary bias, and also to study test of hypothesis and the theoretical behavior of estimators for stationary lagged time series. Moreover, the study of a multivariate local version of the global measures of Kendall's tau and Blomqvist's beta can also be interesting.

\section{Acknowledgements}

This work was partially supported by FAPESP grants 2008/51097-6 and 2013/00506-1. The authors are grateful to two referees, whose comments considerably improved the presentation of the paper. 


\section{References}

Azzalini, A. (1981). A note on the estimation of a distribution function and quantiles by a kernel method. Biometrika, 68(1), 326-328. http://dx.doi.org/10.1093/biomet/68.1.326

Bairamov, I., Kotz, S., \& Kozubowski, T. J. (2003). A new measure of linear local dependence. Statistics, 37(3), 243-258. http://dx.doi.org/10.1080/0233188021000011839

Bjerve, S., \& Doksum, K. (1993). Correlation curves: Measures of association as functions of covariate values. Annals of Statistics, 21(2), 890-902. http://dx.doi.org/10.1214/aos/1176349156

Dhaene, J., Linders, D., Schoutens, W., \& Vyncke, D. (2013). A multivariate dependence measure for aggregating risks. Technical report. Leuven: Faculty of Economics and Business.

Fermanian, J. D., Radulovic, D., \& Wegkamp, M. (2004). Weak convergence of empirical copula processes. Bernoulli, 10(5), 847-860. http://dx.doi.org/10.3150/bj/1099579158

Fermanian, J.-D., \& Scaillet, O. (2003). Nonparametric estimation of copulas for time series. Journal of Risk, 5, 25-54.

Fisher, N. I. (1997). Copulas. In Encyclopedia of Statistical Sciences (Vol. 1, pp. 159-163). New York: John Wiley \& Sons.

GänBler, P., \& Stute, W. (1987). Seminar on empirical processes, DMV-Seminar (Vol. 9). Basel: Birkhäuser Verlag.

Joe, H. (1989). Relative Entropy Measures of Multivariate Dependence. Journal of the American Statistical Association, 84(405), 157-164. http://dx.doi.org/10.1080/01621459.1989.10478751

Joe, H. (1990). Multivariate concordance. Journal of Multivariate Analysis, 35, 12-30. http://dx.doi.org/10.1016/0047-259X(90)90013-8

Joe, H. (1997). Multivariate Models and Dependence Concepts. Boca Raton: Chapman \& Hall/CRC. http://dx.doi.org/10.1201/b13150

Nelsen, R. B. (1996). Nonparametric measures of multivariate association. Distribution with Fixed Marginals and Related Topics, 28, 223-232. IMS Lecture Notes: Monograph Series.

Nelsen, R. B. (2002). Concordance and copulas: A survey. In C. M. Cuadras, J. Fortiana, \& J. A. RodriguezLallena (Eds.), Distributions with Given Marginals and Statistical Modelling (pp. 169-177). Dordrecht: Kluwer Academic Publishers. http://dx.doi.org/10.1007/978-94-017-0061-0_18

Nelsen, R. B. (2006). An Introduction to Copulas (2nd ed.). New York: Springer-Verlag.

Nešlehová, J. (2004). Dependence of non-continuous random variables. Ph. D. Thesis. Carl von Ossietzky Universität Oldenburg, Shaker Verlag, Aachen.

Rüchendorf, L. (1976). Asymptotic normality of multivariate rank order statistics. Annals of Statistics, 4, $912-923$. http://dx.doi.org/10.1214/aos/1176343588

Ruymgaart, F. H., \& van Zuijlen, M. C. A. (1976). Asymptotic normality of multivariate linear rank statistics in the non-i.i.d. case. Annals Statistics, 6(3), 588-602. http://dx.doi.org/10.1214/aos/1176344203

Schmid, F., \& Schmidt, R. (2007a). Multivariate extensions of Spearman's rho and related statistics. Statistics \& Probability Letters, 77, 407-416.

Schmid, F., \& Schmidt, R. (2007b). Multivariate conditional versions of Spearman's rho and related measures of tail dependence. Journal of Multivariate Analysis, 98, 1123-1140. http://dx.doi.org/10.1016/j.jmva.2006.05.005

Segers, J. (2012). Asymptotics of empirical copula processes under non-restrictive smoothness assumptions. Bernoulli, 18(3), 764-782. http://dx.doi.org/10.3150/11-BEJ387

Sibuya, M. (1960). Bivariate Extreme Statistics. Annals of the Institute of Statistical Mathematics, 11, $195-210$. http://dx.doi.org/10.1007/BF01682329

Sklar, A. (1959). Fonctions de répartition à n dimensions e leurs marges. Publications de l'Institut de Statistique de l'Université de Paris, 8, 229-231. 
Sricharan, K., Hero, A. O., \& Rajaratnam, B. (2011). A local dependence measure and its application to screening for high correlations in large data sets. Proceedings of the 14th International Conference on Information Fusion, 1-8.

Stute, W. (1984). The oscillation behavior of empirical processes: the multivariate case. Annals of Probability, 12, 361-379. http://dx.doi.org/10.1214/aop/1176993295

Tasena, S., \& Dhompongsa, S. (2013). A measure of multivariate mutual complete dependence. International Journal of Approximate Reasoning, 54(6), 748-761. http://dx.doi.org/10.1016/j.ijar.2013.01.001

Taylor, M. D. (2007). Multivariate measures of concordance. Annals of the Institute for Statistical Mathematics, 59(4), 789-806. http://dx.doi.org/10.1007/s10463-006-0076-2

Tjøstheim, D., \& Hufthammer, K. O. (2013). Local Gaussian correlation: a new measure of dependence. Journal of Econometrics, 172(1), 33-48. http://dx.doi.org/10.1016/j.jeconom.2012.08.001

Tsukuhara, H. (2005). Semiparametric estimation in copula models. Canadian Journal Statistics, 33(3), $357-375$. http://dx.doi.org/10.1002/cjs.5540330304

Úbeda-Flores, M. (2005). Multivariate versions of Blomqvist's beta and Spearman's footrule. Annals of the Institute of Statistical Mathematics, 57, 781-788. http://dx.doi.org/10.1007/BF02915438

van der Vaart, A. W. \& Wellner, J. A. (1996). Weak Convergence and Empirical Process. New York: SpringerVerlag. http://dx.doi.org/10.1007/978-1-4757-2545-2

Wolff, E. F. (1980). N-dimensional measures of dependence. Stochastica, 4(3), 175-188.

\section{Copyrights}

Copyright for this article is retained by the author(s), with first publication rights granted to the journal.

This is an open-access article distributed under the terms and conditions of the Creative Commons Attribution license (http://creativecommons.org/licenses/by/3.0/). 